\title{
Role of the neuromuscular ultrasound in the diagnostic of the multifocal motor neuropathy
}

\author{
Antonios Kerasnoudis ${ }^{1}$, Kalliopi Pitarokoili², Min-Suk Yoon² \\ ${ }^{1}$ Department of Neuroimmunology, St. Luke Hospital, 55236 Panorama, Thessaloniki, Greece. \\ ${ }^{2}$ Department of Neurology, St. Josef Hospital, Ruhr-University, 44791 Bochum, North Rhine-Westphalia, Germany.
}

\section{A B S T R A C T}

Multifocal motor neuropathy ( $\mathrm{MMN}$ ) is the one of the most common acquired immune-mediated inflammatory disorders of the peripheral nervous system. The diagnosis is based on the distribution pattern of the neurological semiology and the pathological changes of nerve conduction studies (NCS) in classical cases. However, in cases with subtle clinical presentation, an extended diagnostic workup may be needed, such as cerebrospinal fluid examination, laboratory tests, and nerve biopsy. NCS remain nowadays fundamental not only for the diagnosis, but also for the follow-up and measurement of response to immune-treatment in MMN. New challenges arose though, on how best to acquire a static and dynamic imaging of the peripheral nerves, aiming to provide a holistic approach to the nerve impairment. According to the literature, neuromuscular ultrasound is able to detect in MMN patients thickened or swollen cervical roots, peripheral nerves or brachial plexus, findings that suggest ongoing inflammation. This review provides a timely update on the nerve ultrasound findings in MMN.

Key words: Brachial plexus, conduction block, immune-mediated neuropathies, multifocal motor neuropathy, nerve hypertrophy, nerve ultrasound

\section{INTRODUCTION}

Multifocal motor neuropathy ( $\mathrm{MMN}$ ) is an intriguing peripheral nerve disease with a prevalence of 1-2/100,000 adults. ${ }^{[1]}$ Several diagnostic criteria have been proposed, mainly summarizing the slowly progressive, asymmetric weakness, with a striking predilection for the upper extremities, whereas sensory fibers and upper motor neuron involvement fail in the disease course ${ }^{[2]}$ Although the detection of conduction block remains the electrophysiological hallmark of the disease, it is important to recognize that it may not be possible to demonstrate this finding even after careful studies, because blocks may be activity-dependent, and the site of pathology may be very proximal in the brachial plexus or nerve root level. ${ }^{[2-5]}$

The first papers defined conduction block as a $20-30 \%$ amplitude or area reduction in the distal compound muscle action potential (CMAP) if the

\begin{tabular}{|l|l|}
\hline \multicolumn{2}{|c|}{ Access this article online } \\
\hline Quick Response Code: & \\
\hline & Website: \\
\hline & www.nnjournal.net \\
& \\
\hline
\end{tabular}

CMAP duration did not exceed 15\% greater than normal. Computer modeling of conduction block and temporal dispersion in an animal model has demonstrated that up to $50 \%$ area reduction of the proximal to distal CMAP can be due entirely to interphase cancellation. Similar studies in human have shown that distal CMAP duration and proximal CMAP duration prolongation are important factors for the definition of conduction block in the median nerve segment over the forearm: the shorter the distal duration and proximal duration prolongation the less CMAP amplitude reduction is needed to diagnose a conduction block. ${ }^{[2]}$ The association between MMN and immunoglobulin M (IgM) antiganglioside GM1 (anti-GM1) antibodies have already been suggested in the literature, however, the diagnostic accuracy of anti-GM1 testing in diagnosing $\mathrm{MMN}$ is unclear. The literature reports the presence of anti-GM1 IgM antibodies in between $30 \%$ and $80 \%$ of MMN patients. ${ }^{[2]}$

Meanwhile, neuromuscular ultrasound is an easily applicable and safe method for studying structural changes in peripheral nerves. Various ultrasound studies have reported pathological ultrasound changes in MMN patients, reporting consistently an asymmetric, inhomogenous increase of the nerve cross-sectional area (CSA). ${ }^{[6-9]}$ Three studies have

Corresponding Author: Dr. Antonios Kerasnoudis, Department of Neuroimmunology, St. Luke Hospital, 55236 Panorama, Thessaloniki, Greece. E-mail: antonis.kerasnoudis@gmail.com 
reported controversial findings on the correlation between sonographic and electrophysiological results in MMN patients. ${ }^{[6,8,10]}$ In view of the severe functional disability of MMN patients, it remains unknown that of these two methods could better highlight the functional and clinical status of these patients. ${ }^{[11]}$

The aim of this review is to provide a timely update on the role of the neuromuscular ultrasound in the diagnostic of the MMN.

\section{QUANTIFICATION OF NERVE ULTRASOUND FINDINGS}

Cross-sectional area reference values for peripheral nerves and brachial plexus have been already reported in the literature. ${ }^{[7,12,13]}$ The difficulty, however, to differentiate normal from a pathologic heterogeneity of CSA changes in peripheral nerves, especially in cases of immune-mediated neuropathies, remains an important limitation of the neuromuscular ultrasound in clinical application. CSA enlargement can be the result either of edema (usually accompanied by disturbed fascicular echostructure) or hypertrophy (usually accompanied by preserved fascisular echostructure).

Novel ultrasound measures, aiming to quantify pathologic ultrasound changes of peripheral nerves in immune-mediated polyneuropathies, have been recently introduced in the literature:[7,9,11-13] (1) the intranerve CSA variability (for each nerve), defined as maximal CSA/minimal CSA; (2) the internerve CSA variability (for each patient), defined as nerve with maximal intranerve CSA variability/nerve with minimal intranerve CSA variability; (3) the side to side difference ratio of the intranerve CSA variability (for each nerve), defined as side with maximal intranerve CSA variability/side with minimal intranerve CSA variability; and (4) the intraplexus CSA variability defined as maximal CSA of the brachial plexus/minimal CSA of the brachial plexus [Table 1].

\begin{tabular}{ll}
\hline Table I: Equations for calculating the intranerve-, \\
internerve-, intraplexus CSA variability and side to side \\
difference ratio of the intranerve CSA variability \\
\hline Variability & Calculating equation \\
\hline $\begin{array}{l}\text { Intranerve CSA variability } \\
\text { (for each nerve) }\end{array}$ & Maximal CSA/minimal CSA \\
$\begin{array}{l}\text { Intranerve CSA variability } \\
\text { (for each subject) }\end{array}$ & $\begin{array}{l}\text { Peripheral nerve with the maximal } \\
\text { intranerve CSA variability/ } \\
\text { peripheral nerve with the minimal } \\
\text { intranerve CSA variability }\end{array}$ \\
$\begin{array}{l}\text { Side to side difference ratio of } \\
\text { the intranerve CSA variability } \\
\text { (for each nerve) } \\
\text { Intraplexus CSA variability } \\
\text { (for each brachial plexus) }\end{array}$ & $\begin{array}{l}\text { CSA variability/side with the } \\
\text { minimal intranerve CSA variability }\end{array}$ \\
\hline CSA: Cross sectional area & $\begin{array}{l}\text { Maximal CSA of brachial plexus/ } \\
\text { minimal CSA of brachial plexus }\end{array}$ \\
\hline
\end{tabular}

CSA: Cross sectional area
Using the intranerve CSA variability, the sonographer may differentiate a focal (higher values) from diffuse (lower values) nerve hypertrophy while the internerve CSA variability may reveal possible distribution patterns of peripheral nerve impairment. ${ }^{[7]}$ On the other hand, the side to side difference ratio of the intranerve CSA variability may be useful in detecting any lateralization of pathologic changes and the intraplexus CSA variability in differentiating focal (higher values) from diffuse (lower values) brachial plexus hypertrophy. ${ }^{[9,13]}$

\section{RESULTS}

Currently, 6 studies (evaluating a total of 55 cases) of nerve sonography in MMN patients have been published [Table 2]..$^{[6-10,14]}$ The first description of pathological ultrasound findings in MMN was published by Beekman et al. ${ }^{[6]}$ In this report, the authors documented at least one anatomical site with pathological hypertrophy of the median or ulnar or radial nerves and/or brachial plexus in $90 \%$ of the cases. The authors concluded that the neuromuscular ultrasound may allow the detection of pathological signs to a greater extent than nerve conduction tests in MMN. In a later study of $12 \mathrm{MMN}$ patients, nerve hypertrophy was documented in the median (forearm), ulnar (Guyons' canal, forearm, elbow, upper arm) and tibial nerve (ankle), but not in brachial plexus, when compared to controls [Figures 1 and 2].

Considering the morphology of peripheral nerve hypertrophy (focal vs. diffuse), Padua et al..$^{[7]}$ have reported the inhomogenous CSA enlargement, mainly of the median, ulnar and fibular nerve in a small group of MMN patients. A second study on two MMN patients not only confirmed the focal type of CSA enlargement, but also documented the significant lateralization of ultrasound findings. ${ }^{\left[{ }^{[8]}\right.}$ Another MMN study has documented a focal type of CSA enlargement in the median nerve, when compared with controls. In addition, the higher values of the internerve CSA variability and "side to side difference ratio of the intranerve CSA variability" of the median, ulnar and fibular nerve, were attributed by the authors to the possible striking predilection of MMN to certain peripheral nerves and the asymmetry of findings respectively. ${ }^{[10]}$

A possible explanation for the CSA enlargement in MMN cases could derive from pathological studies at sites of conduction blocks. According to these studies, perivascular areas contain scattered demyelinated axons, which are often surrounded by small onion bulb formations. ${ }^{[15]}$ These onion bulb formation may lead to a consecutive CSA enlargement of the nerve. In addition, pathological CSA changes are usually detected at 
Table 2: An overview of the existing nerve ultrasound studies on MMN and their pathological findings

\begin{tabular}{|c|c|c|c|c|c|c|c|c|c|}
\hline Authors & $\begin{array}{l}\text { Patients } \\
\text { (n) }\end{array}$ & $\begin{array}{l}\text { Controls } \\
(n)\end{array}$ & $\begin{array}{l}\text { Median } \\
\text { nerve }\end{array}$ & $\begin{array}{l}\text { Ulnar } \\
\text { nerve }\end{array}$ & $\begin{array}{l}\text { Brachial plexus } \\
\text { or cervical roots }\end{array}$ & $\begin{array}{l}\text { Sciatic } \\
\text { nerve }\end{array}$ & $\begin{array}{c}\text { Femoral } \\
\text { nerve }\end{array}$ & $\begin{array}{l}\text { Fibular } \\
\text { nerve }\end{array}$ & $\begin{array}{l}\text { Tibial } \\
\text { nerve }\end{array}$ \\
\hline Beekman et al. ${ }^{[6]}$ & 21 & 20 & $\mathrm{x}$ & $x$ & $\mathrm{x}$ & - & - & - & - \\
\hline Padua et al..$^{[7]}$ & 2 & 63 & $\mathrm{x}$ & $x$ & - & - & - & $x$ & - \\
\hline Kerasnoudis ${ }^{[8]}$ & 2 & 30 & $\mathrm{x}$ & $x$ & - & - & - & $x$ & - \\
\hline Kerasnoudis et al..$^{[9]}$ & 1 & - & $\mathrm{x}$ & $\mathrm{N}$ & $\mathrm{N}$ & - & - & - & - \\
\hline Kerasnoudis et al. ${ }^{[10]}$ & 12 & 80 & $x$ & $x$ & $\mathrm{~N}$ & - & - & $\mathrm{N}$ & $x$ \\
\hline Zaidman et al. ${ }^{[14]}$ & 17 & - & $\hat{x}$ & $x$ & - & - & - & - & - \\
\hline
\end{tabular}

MMN: Multifocal motor neuropathy; $\mathrm{x}$ : The concrete nerve was reported with pathological findings; $\mathrm{N}$ : The concrete nerve was reported with normal findings; -: The concrete nerve was not studied at all; $n$ : Absolute number
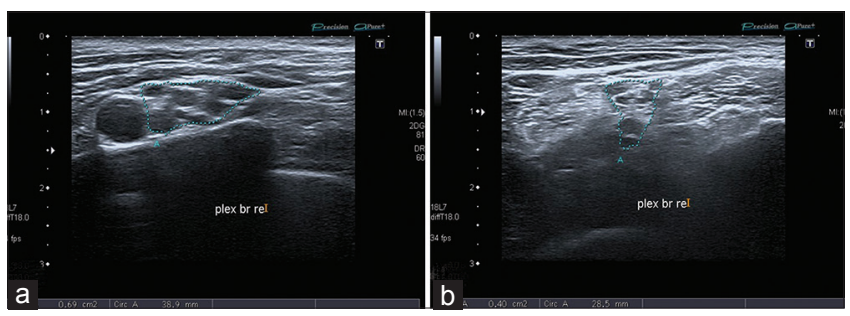

Figure 1: Axial scan of the brachial plexus in a multifocal motor neuropathy (MMN) patient in supraclavicular (a) and interscalene space (b) in a MMN patient. In this case both the cross sectional area and the intraplexus cross sectional area variability of the brachial plexus are within the reference values of our lab ${ }^{[13]}$

several proximal and distal sites in the anatomic course of the peripheral nerves in MMN patients. This finding may reflect the immune-mediated patchy multifocal demyelination occurring along the motor nerve fibers in this type of immune-mediated injury. ${ }^{[10,11]}$

Another important aspect in the field of sonography in MMN is the possible use of this method for identifying nerve conduction blocks. The localization of the nerve conduction block is often difficult to be identified in the nerve conduction studies (NCS), especially when dealing with proximal parts of the nerves. By overlooking the electrophysiological hallmark of the disease, delay in the diagnosis and therefore delayed treatment can occur. ${ }^{[2]}$ Beekman et al. ${ }^{[6]}$ documented pathological ultrasound findings not only at sites with electrophysiological impairment, but also at sites with normal functioning in NCS. An absolute correlation between site of nerve hypertrophy and site of conduction block has been reported only in one case in the literature. ${ }^{[8]}$ Another study on $12 \mathrm{MMN}$ patients showed a significant correlation between sonographic and electrophysiological findings only between the CMAP and CSA of the median nerve at the upper arm. ${ }^{[10]}$ Systematic prospective studies on the sensitivity of ultrasound in detecting focal immune-mediated nerve lesions fail in the literature.

An interesting point of future study is the applicability of the nerve ultrasound as screening method for immune-therapy in dysimmune neuropathies. Nerve ultrasound and NCS failed to highlight functional disability in post-Guillain-Barré syndrome and chronic inflammatory demyelinating polyneuropathy patients
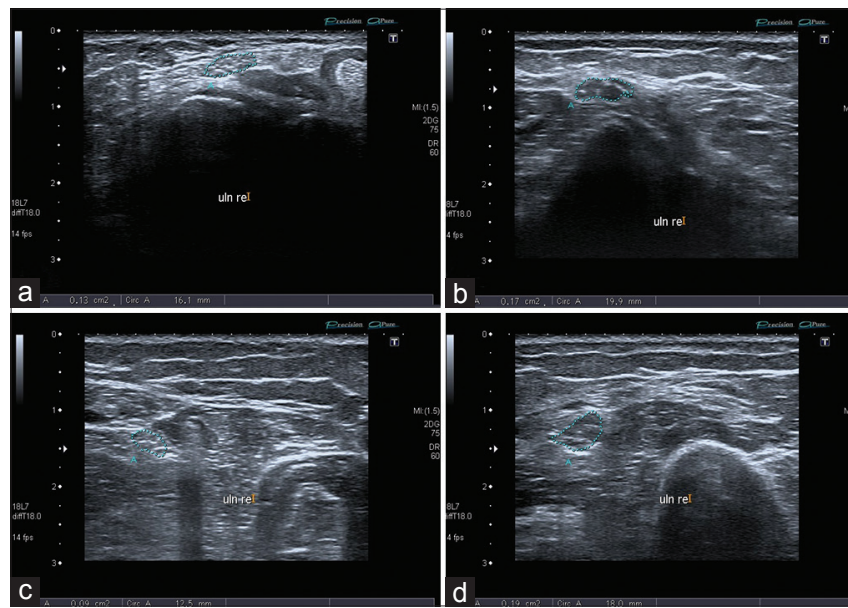

Figure 2: Axial scan of the ulnar nerve in a multifocal motor neuropathy (MMN) patient in Guyon's canal (a), forearm (b), elbow (c) and upper-arm (d) showing a pathological cross sectional area enlargement at all anatomic sites, when compared to the reference values of our lab. ${ }^{[13]}$ This finding may reflect the immune-mediated patchy multifocal demyelination occurring in MMN

in the literature. ${ }^{[16,17]}$ In a later study on MMN patients, neither sonography nor electrophysiology correlated with the Medical Research Council sum score, Rasch-built Overall Disability Scale score or Rasch-built fatigue severity scale. ${ }^{[10]}$ These studies have shown that the already known ultrasound biomarkers (CSA, echogenity, intranerve CSA variability) are not able to highlight the effectivity of immune-therapy. ${ }^{[1]}$

\section{CONCLUSION}

To summarize, the currently available ultrasound studies show that mainly a focal type of asymmetrical peripheral nerve enlargement is expected in MMN. Nerve ultrasound findings seem to show no significant correlation to electrophysiological findings at most anatomical sites. In addition, prospective studies on the applicability of ultrasound as screening method of immune-therapy fail in the literature, while various retrospective studies failed to highlight any significant correlation between ultrasound findings and functional disability.

As the main uncertainties regarding the diagnostic criteria of MMN are steadily resolved, new challenges continuously arise on how to acquire the best static and dynamic imaging of the relevant nerve structures 
in this type of immune-mediated disease, aiming to provide a complementary and holistic approach to nerve impairment. The first nerve ultrasound studies on MMN have shown that ultrasound seems to be a reliable and easily applicable method to detect pathological structural changes in peripheral nerves. The quantification of ultrasound changes and highlighting the distribution patterns of pathological findings remains a challenging aspect of future study. The recently proposed measurements in the literature may help to achieve this goal, but multicentre prospective validation is needed..$^{[7,8,12,13]}$

\section{REFERENCES}

1. Mahdi-Rogers M, Hughes RA. Epidemiology of chronic inflammatory neuropathies in southeast England. Eur J Neurol 2014;21:28-33.

2. Joint Task Force of the EFNS and the PNS. European Federation of Neurological Societies/Peripheral Nerve Society guideline on management of multifocal motor neuropathy. Report of a joint task force of the European Federation of Neurological Societies and the Peripheral Nerve Society - first revision. J Peripher Nerv Syst 2010;15:295-301.

3. Nodera H, Bostock H, Izumi Y, Nakamura K, Urushihara R, Sakamoto T, Murase N, Shimazu H, Kusunoki S, Kaji R. Activity-dependent conduction block in multifocal motor neuropathy: magnetic fatigue test. Neurology 2006;67:280-7.

4. Pakiam AS, Parry GJ. Multifocal motor neuropathy without overt conduction block. Muscle Nerve 1998;21:243-5.

5. Delmont E, Azulay JP, Giorgi R, Attarian S, Verschueren A, Uzenot D, Pouget J. Multifocal motor neuropathy with and without conduction block: a single entity? Neurology 2006;67:592-6.

6. Beekman R, van den Berg LH, Franssen H, Visser LH, van Asseldonk JT, Wokke JH. Ultrasonography shows extensive nerve enlargements in multifocal motor neuropathy. Neurology 2005;65:305-7.

7. Padua L, Martinoli C, Pazzaglia C, Lucchetta M, Granata G, Erra C, Briani C. Intra-and internerve cross-sectional area variability: new ultrasound measures. Muscle Nerve 2012;45:730-3.
8. Kerasnoudis A. Correlation of sonographic and electrophysiological findings in a patient with multifocal motor neuropathy. J Neuroimaging 2014;24:305-7.

9. Kerasnoudis A, Klasing A, Behrendt V, Gold R, Yoon MS. Intra-and internerve cross-sectional area variability: New ultrasound measures. Muscle Nerve 2013;47:146-7.

10. Kerasnoudis A, Pitarokoili K, Behrendt V, Gold R, Yoon MS. Multifocal motor neuropathy: correlation of nerve ultrasound, electrophysiological and clinical findings. J Peripher Nerv Syst 2014;19:165-74

11. Kerasnoudis A, Yoon MS. The role of neuromuscular ultrasound in the diagnostic of immune-mediated neuropathies. In: Brown MO, Budd BA, editors. Advances in Neuropathy Research. New York: Nova Science Publishers; 2013. p. 119-33.

12. Kerasnoudis A, Pitarokoili K. Ulnar nerve reference values for cross-sectional area, intranerve cross sectional area variability and side to side difference ratio. Rheumatol Int 2014;34:551-2.

13. Kerasnoudis A, Pitarokoili K, Behrendt V, Gold R, Yoon MS. Cross sectional area reference values for sonography of peripheral nerves and brachial plexus. Clin Neurophysiol 2013;124:1881-8.

14. Zaidman CM, Harms MB, Pestronk A. Ultrasound of inherited vs. acquired demyelinating polyneuropathies. J Neurol 2013;260:3115-21.

15. Kaji R, Oka N, Tsuji T, Mezaki T, Nishio T, Akiguchi I, Kimura J. Pathological findings at the site of conduction block in multifocal motor neuropathy. Ann Neurol 1993;33:152-8.

16. Kerasnoudis A, Pitarokoili K, Behrendt V, Gold R, Yoon MS. Correlation of nerve ultrasound, electrophysiological and clinical findings in chronic inflammatory demyelinating polyneuropathy. $J$ Neuroimaging 2014. doi: 10.1111/jon. 12079.

17. Kerasnoudis A, Pitarokoili K, Behrendt V, Gold R, Yoon MS. Correlation of nerve ultrasound, electrophysiological, and clinical findings in post Guillain-Barré syndrome. J Peripher Nerv Syst 2013;18:232-40.

Cite this article as: Kerasnoudis A, Pitarokoili K, Yoon MS. Role of the neuromuscular ultrasound in the diagnostic of the multifocal motor neuropathy. Neuroimmunol Neuroinflammation 2014;1(3):103-6.

Source of Support: Nil. Conflict of Interest: No.

Received: 08-07-2014; Accepted: 21-08-2014 